\title{
NORM OF SCHUR MULTIPLICATION FOR SCHATTEN NORM
}

\author{
K. OKUBO
}

(Communicated by Louis J. Ratliff, Jr.)

\begin{abstract}
Let $\mathbb{M}_{n}$ denote the algebra of all $n \times n$ complex matrices and $\|\cdot\|_{p}$ be the Schatten's $p$-norm on $\mathbb{M}_{n}$. For each $A \in \mathbb{M}_{n}$, a linear operator $S_{A}$ on $\mathbb{M}_{n}$ is defined by $S_{A}(X):=A \circ X$ for all $X \in \mathbb{M}_{n}$, where $\circ$ denotes the Schur product and $\left\|S_{A}\right\|_{p, q}$ is defined as the operator norm from $\left(\mathbb{M}_{n},\|\cdot\|_{p}\right)$
\end{abstract} to $\left(\mathbb{M}_{n},\|\cdot\|_{q}\right)$ for $p, q \geq 1$.

Given an $A \in \mathbb{M}_{n}$, suppose $0<\lambda<1$, and $p, p_{1}, p_{2}, q, q_{1}$, and $q_{2}$ are not smaller than 1 , and

$$
\frac{1}{p}=\frac{\lambda}{p_{1}}+\frac{1-\lambda}{p_{2}} \text { and } \frac{1}{q}=\frac{\lambda}{q_{1}}+\frac{1-\lambda}{q_{2}}
$$

are satisfied. Then we will show that $\left\|S_{A}\right\|_{p, q} \leq\left\|S_{A}\right\|_{p_{1}, q_{1}}^{\lambda} \cdot\left\|S_{A}\right\|_{p_{2}, q_{2}}^{1-\lambda}$.

\section{INTRODUCTION AND NOTATIONS}

Let $\mathbb{M}_{n}$ be the algebra of all $n \times n$ complex matrices. The Schur product (also called Hadamard product) $A \circ B$ of two matrices $A=\left(a_{i j}\right)$ and $B=\left(b_{i j}\right)$ in $\mathbb{M}_{n}$ is defined by

$$
A \circ B:=\left(a_{i j} \cdot b_{i j}\right),
$$

and for given $A \in \mathbb{M}_{n}$, the Schur multiplication operator $S_{A}$ on $\mathbb{M}_{n}$ is defined by

$$
S_{A}(X):=A \circ X \text { for all } X \in \mathbb{M}_{n} \text {. }
$$

We denote the singular values of $A \in \mathbb{M}_{n}$, arranged in nonincreasing order, by $s_{1}(A) \geq s_{2}(A) \geq \cdots \geq s_{n}(A)$; that is, $s_{i}(A)$ is the $i$ th largest (with multiplicities counted) eigenvalue of $\left(A^{*} A\right)^{1 / 2}$. For $A \in \mathbb{M}_{n}$ and $p \geq 1$, the Schatten's p-norm $\|A\|_{p}$ of $A$ is defined by

$$
\|A\|_{p}:=\left(\sum_{i=1}^{n} s_{i}^{p}(A)\right)^{1 / p} .
$$

Received by the editors November 20, 1989 and, in revised form, March 7, 1990; presented at the ILAS Inaugural Meeting, International Linear Algebra Society, Provo, Utah, August, 1989.

1980 Mathematics Subject Classification (1985 Revision). Primary 15A60, 15A04, 47A30.

Key words and phrases. Schur multiplication, Schatten norm. 
In particular, when $p=1,2$ and $\infty$, norm $\|\cdot\|_{p}$ is called trace norm, Frobenius norm, and spectral norm, respectively.

We denote the operator norm of $S_{A}$ from $\left(\mathbb{M}_{n},\|\cdot\|_{p}\right)$ to $\left(\mathbb{M}_{n},\|\cdot\|_{q}\right)$ by $\left\|S_{A}\right\|_{p, q}$, i.e.,

$$
\left\|S_{A}\right\|_{p, q}=\sup _{B \in \mathbb{M}_{n}}\left\|S_{A}(B)\right\|_{q} /\|B\|_{p},
$$

and we write $\left\|S_{A}\right\|_{p} \equiv\left\|S_{A}\right\|_{p, p}$.

A norm $\|\cdot\|$ is called unitary invariant if $\|A\|=\|U A V\|$ for all $A \in \mathbb{M}_{n}$ and all unitary $U, V \in \mathbb{M}_{n}$. For arbitrary $p \geq 1,\|\cdot\|_{p}$ is an example of a unitarily invariant norm.

Given a norm $\|\cdot\|$ on $\mathbb{M}_{n}$, under the coupling $\langle X, Y\rangle \equiv \operatorname{tr}\left(X Y^{*}\right)$ for $X, Y \in \mathbb{M}_{n}$ the dual norm $\|\cdot\|^{D}$ (on $\mathbb{M}_{n}$ ) of $\|\cdot\|$ is defined by

$$
\|A\|^{D}:=\sup _{B \in \mathbb{M}_{n}}\left|\operatorname{tr}\left(A B^{*}\right)\right| /\|B\| \text {. }
$$

Then it is known (see [2, pp. 130-135]) that $\|\cdot\|_{q}$ becomes the dual norm of $\|\cdot\|_{p}$ for $p, q \geq 1$ such that $\frac{1}{p}+\frac{1}{q}=1$.

Since the dual map of $S_{A}$ on the Banach space $\left(\mathbb{M}_{n},\|\cdot\|_{q}\right)$ is given as $S_{\bar{A}}$ on $\left(\mathbb{M}_{n},\|\cdot\|_{q}\right)$ where $\bar{A}$ is the complex conjugation of $A$, it is easy to show $\left\|S_{A}\right\|_{p}=\left\|S_{A}\right\|_{q}$ if $p, q \geq 1$ and $\frac{1}{p}+\frac{1}{q}=1$. We shall obtain some convex property of the function $p \rightarrow\left\|S_{A}\right\|_{p}(p \geq 1)$ for given $A \in \mathbb{M}_{n}$.

In the sequel, for a row vector $\mathfrak{x}=\left(\xi_{1}, \xi_{2}, \ldots, \xi_{n}\right)$ of $n$-tuple of complex numbers, ${ }^{t} \mathfrak{x}$ denotes the transpose of $\mathfrak{x}$, that is, ${ }^{t} \mathfrak{x}$ is a column vector such that

$$
{ }^{t} \mathfrak{x}=\left(\begin{array}{c}
\xi_{1} \\
\xi_{2} \\
\vdots \\
\xi_{n}
\end{array}\right)
$$

\section{RESULTS AND REMARKS}

First of all, we may mention the known results of norm $S_{A}$.

$$
\|A \circ B\|_{\infty} \leq\|A\|_{\infty} \cdot\|B\|_{\infty} \quad \text { for all } A, B \in \mathbb{M}_{n} \cdot \quad \text { (Schur [7]) }
$$

This Schur's result implies

$$
\left\|S_{A}\right\|_{\infty} \leq\|A\|_{\infty} \quad\left(A \in \mathbb{M}_{n}\right) .
$$

We denote the Euclidean norms of the columns of $A$, arranged in nonincreasing order, by $c_{1}(A) \geq c_{2}(A) \geq \cdots \geq c_{n}(A)$ and the Euclidean norms of the rows of $A$ by $r_{1}(A) \geq r_{2}(A) \geq \cdots \geq r_{n}(A)$. For $A \in \mathbb{M}_{n}$ and $\alpha \in(0,1)$, we write $t_{i}(A, \alpha)=\left\{p_{i}(A, \alpha) \cdot q_{i}(A, 1-\alpha)\right\}^{1 / 2}$ where $p_{i}(A, \alpha)$ is the $i$ th largest main diagonal entry of $\left(A A^{*}\right)^{\alpha}$ and $q_{i}(A, 1-\alpha)$ is the $i$ th largest main diagonal entry of $\left(A^{*} A\right)^{1-\alpha}$.

$$
\left\|S_{A}\right\|_{\infty} \leq \min \left\{r_{1}(A), c_{1}(A)\right\} \quad \text { for all } A \in \mathbb{M}_{n} \text {. (Ong [5]) }
$$


(9) $\left\|S_{A}\right\|_{\infty} \leq t_{1}(A, \alpha)$ for all $A \in \mathbb{M}_{n}$ and $\alpha \in(0,1)$. (Walter [8])

Next, in (10) and (11) we consider a unitarily invariant norm $\|\cdot\|_{\mu}$ and operator norm of $S_{A}$ with respect to $\|\cdot\|_{\mu}$; that is,

$$
\left\|S_{A}\right\|_{\mu}:=\sup _{B \in \mathbb{M}_{n}}\left\|S_{A}(B)\right\|_{\mu} /\|B\|_{\mu} .
$$

Let $\|\cdot\|_{\mu}$ be a unitarily invariant norm on $\mathbb{M}_{n}$. Then

$$
\|A \circ B\|_{\mu} \leq\|A\|_{\infty} \cdot\|B\|_{\mu} \text { for all } A, B \in \mathbb{M}_{n} \text {. (Okubo [4]) }
$$

We obtain from this result

$$
\left\|S_{A}\right\|_{\mu} \leq\|A\|_{\infty} \text { for all } A \in \mathbb{M}_{n}
$$

Let $\|\cdot\|_{\mu}$ be a unitarily invariant norm on $\mathbb{M}_{n}$. Then

$$
\begin{array}{r}
\left\|S_{A}\right\|_{\mu} \leq \inf \left\{c_{1}(X) \cdot c_{1}(Y) \mid X, Y \in \mathbb{M}_{r, n} ; X^{*} Y=A, r \geq 1\right\} \quad \text { for all } A \in \mathbb{M}_{n} . \\
\text { (Ando, Hern, and Johnson [1]) }
\end{array}
$$

Haagerup (cf. [6]) showed that the value of the right-hand side of (13) is equal to $\left\|S_{A}\right\|_{\infty}$; hence we have

$$
\left\|S_{A}\right\|_{\mu} \leq\left\|S_{A}\right\|_{\infty} \text { for all } A \in \mathbb{M}_{n} .
$$

Proposition 1. If $p \geq 1$, then

$$
\left\|S_{A}\right\|_{2} \leq\left\|S_{A}\right\|_{p} \text { for all } A \in \mathbb{M}_{n} .
$$

Proof. Let $A=\left(a_{i j}\right)$. It is easy to show that

$$
\left\|S_{A}\right\|_{2}=\max _{1 \leq i, j \leq n}\left|a_{i j}\right| \text {. }
$$

(This is mentioned by Ando, Horn, and Johnson in [1].) Let

$$
e_{i}={ }^{t}(0, \ldots, 0, \stackrel{(i)}{1}, 0, \ldots, 0) \text { for } i=1,2, \ldots, n .
$$

Since $S_{A}\left(E_{i j}\right)=a_{i j} E_{i j}$ where $E_{i j}=e_{i} \otimes e_{j}^{*}, a_{i j}$ is an eigenvalue of $S_{A}$ for all $1 \leq i, j \leq n$. It follows from this fact that

$$
\left\|S_{A}\right\| \geq\left\|S_{A}\left(E_{i j}\right)\right\| /\left\|E_{i j}\right\|=\left|a_{i j}\right|
$$

for any norm $\|\cdot\|$ on $\mathbb{M}_{n}$ and all $i, j$ of $1 \leq i, j \leq n$; hence we have, from (17), $\left\|S_{A}\right\|_{2} \leq\left\|S_{A}\right\|_{p}$ for $p \geq 1$.

Remark. It is known that if $A \geq 0$ (nonnegative definite), then

$$
\left\|S_{A}\right\|_{\infty}=\max _{1 \leq i \leq n}\left|a_{i i}\right| \quad \text { where } A=\left(a_{i j}\right) .
$$

Therefore, as the proof of Proposition 1, we obtain that if $A \geq 0$ then $\left\|S_{A}\right\|_{\mu}=$ $\max _{1 \leq i \leq n}\left|a_{i i}\right|$. 
As mentioned before we have $\left\|S_{A}\right\|_{p}=\left\|S_{A}\right\|_{q}$ for $p, q \geq 1$ such that $\frac{1}{p}+\frac{1}{q}=$ 1. In particular, $\left\|S_{A}\right\|_{1}=\left\|S_{A}\right\|_{\infty}$, and from Proposition 1 it follows that

$$
\min _{1 \leq p \leq \infty}\left\|S_{A}\right\|_{p}=\left\|S_{A}\right\|_{2} \text {. }
$$

We have interest in the behavior of the function $[1, \infty] \ni p \rightarrow\left\|S_{A}\right\|_{p}$ for given $A \in \mathbb{M}_{n}$.

To show the main result in this section we need the following lemma obtained by M. Riesz (cf. [3, pp. 214-215]).

Let $A \in \mathbb{M}_{n}$ and $1 \leq s, t \leq \infty$ be given. Then we define $\|A\|_{s, t}$ by (20)

$$
\|A\|_{s, t}:=\sup _{\xi \in C^{n}}\|A \xi\|_{t} /\|\xi\|_{s},
$$

where $\|\xi\|_{s}=\left(\sum_{i=1}^{n}\left|\xi_{i}\right|^{s}\right)^{1 / s}$ for $\xi={ }^{t}\left(\xi_{1}, \xi_{2}, \ldots, \xi_{n}\right)$.

Lemma 2 [M. Riesz]. Let $A \in \mathbb{M}_{n}$ and $\lambda \in(0,1)$ be given. If $s, s_{1}, s_{2}, t, t_{1}, t_{2}$ $\geq 1$ and

then

$$
\frac{1}{s}=\frac{\lambda}{s_{1}}+\frac{1-\lambda}{s_{2}}, \quad \frac{1}{t}=\frac{\lambda}{t_{1}}+\frac{1-\lambda}{t_{2}}
$$

$$
\|A\|_{s, t} \leq\|A\|_{s_{1}, t_{1}}^{\lambda} \cdot\|A\|_{s_{2}, t_{2}}^{1-\lambda} .
$$

Theorem 3. Let $A \in \mathbb{M}_{n}$ and $\lambda \in(0,1)$ be given. If $p, p_{1}, p_{2}, q, q_{1}, q_{2} \geq 1$ and

then

$$
\frac{1}{p}=\frac{\lambda}{p_{1}}+\frac{1-\lambda}{p_{2}}, \quad \frac{1}{q}=\frac{\lambda}{q_{1}}+\frac{1-\lambda}{q_{2}}
$$

$$
\left\|S_{A}\right\|_{p, q} \leq\left\|S_{A}\right\|_{p_{1}, q_{1}}^{\lambda} \cdot\left\|S_{A}\right\|_{p_{2}, q_{2}}^{1-\lambda} .
$$

Proof. By the singular value decomposition of a matrix, $\|B\|_{s} \leq 1$ if and only if $B$ is written by

$$
B=U \Sigma V
$$

where $U, V$ are unitary and $\Sigma=\operatorname{diag}\left(s_{1}(A), s_{2}(A), \ldots, s_{n}(A)\right)$ such that $\sum_{i=1}^{n} s_{i}^{s}(A) \leq 1$. We may assume $t, t^{\prime} \geq 1$ and $\frac{1}{t}+\frac{1}{t^{\prime}}=1$. By the definition of dual norm,

$$
\left\|S_{A}(B)\right\|_{t}=\sup _{\|C\|_{t^{\prime}} \leq 1}\left|\operatorname{tr}(A \circ B) \cdot C^{*}\right| .
$$

From $\|C\|_{t^{\prime}} \leq 1, C$ is written by $C=U^{\prime} \Sigma^{\prime} V^{\prime}$ where $U^{\prime}, V^{\prime}$ are unitary and $\Sigma^{\prime}=\operatorname{diag}\left(s_{1}(C), s_{2}(C), \ldots, s_{n}(C)\right)$ such that $\sum_{i=1}^{n} s_{i}^{t^{\prime}}(C) \leq 1$.

Therefore

$$
\begin{aligned}
\left\|S_{A}\right\|_{s, t} & =\sup _{\|B\|_{s} \leq 1}\left\|S_{A}(B)\right\|_{t} \\
& =\sup _{\|B\|_{s} \leq 1} \sup _{\|C\|_{t^{\prime}} \leq 1}\left|\operatorname{tr}(A \circ B) \cdot C^{*}\right| \\
& =\sup _{\|B\|_{s} \leq 1} \sup _{\|C\|_{t^{\prime}} \leq 1}\left|\operatorname{tr}(A \circ(U \cdot \operatorname{diag}(s(B)) \cdot V)) \cdot V^{\prime *} \cdot \operatorname{diag}(s(C)) \cdot U^{\prime *}\right|
\end{aligned}
$$


where

$$
s(B)={ }^{t}\left(s_{1}(B), s_{2}(B), \ldots, s_{n}(B)\right)
$$

and

$$
s(C)={ }^{t}\left(s_{1}(C), s_{2}(C), \ldots, s_{n}(C)\right) .
$$

Since there exists the matrix $T=T_{U, V, U^{\prime}, V^{\prime}}$ dependent on unitary $U, V$, $U^{\prime}$, and $V^{\prime}$ such that

$$
\begin{aligned}
\langle T(s(B)), s(C)\rangle & =\operatorname{tr}\left((A \circ U \cdot \operatorname{diag}(s(B)) \cdot V) \cdot V^{\prime *} \cdot \operatorname{diag}(s(C)) \cdot U^{\prime *}\right), \\
\left\|S_{A}\right\|_{s, t} & =\sup _{U, V, U^{\prime}, V^{\prime}} \sup _{\sup _{s} \leq 1,\|\eta\|_{t^{\prime} \leq 1}}\left|\left\langle T_{U, V, U^{\prime}, V^{\prime}} \xi, \eta\right\rangle\right| \\
& =\sup _{U, V, U^{\prime}, V^{\prime}}\left\|T_{U, V, U^{\prime}, V^{\prime}}\right\|_{s, t} .
\end{aligned}
$$

Hence, according to Lemma 2 we have

$$
\begin{aligned}
\left\|S_{A}\right\|_{S, t} & \leq \sup _{U, V, U^{\prime}, V^{\prime}}\left\|T_{U, V, U^{\prime}, V^{\prime}}\right\|_{S_{1}, t_{1}}^{\lambda} \cdot\left\|T_{U, V, U^{\prime}, V^{\prime}}\right\|_{S_{2}, t_{2}}^{1-\lambda} \\
& \leq\left\|S_{A}\right\|_{S_{1}, t_{1}}^{\lambda} \cdot\left\|S_{A}\right\|_{S_{2}, t_{2}}^{1-\lambda} \cdot \square
\end{aligned}
$$

Corollary 4. Let $A \in \mathbb{M}_{n}$ be given. Then $\left\|S_{A}\right\|_{p} \leq\left\|S_{A}\right\|_{q}(1 \leq q \leq p \leq 2)$ and $\left\|S_{A}\right\|_{p} \leq\left\|S_{A}\right\|_{q}(2 \leq p \leq q<\infty)$.

Proof. From remark (19), $\min \left\{\left\|S_{A}\right\|_{p} \mid p \geq 1\right\}=\left\|S_{A}\right\|_{2}$, and when $p=q$, $p_{1}=q_{1}$, and $p_{2}=q_{2}$, it follows from Theorem 3 that

$$
\left\|S_{A}\right\|_{p} \leq\left\|S_{A}\right\|_{p_{1}}^{\lambda} \cdot\left\|S_{A}\right\|_{p_{2}}^{1-\lambda} \quad\left(A \in \mathbb{M}_{n}\right)
$$

for $p, p_{1}, p_{2} \geq 1$ and $\frac{1}{p}=\frac{\lambda}{p_{1}}+\frac{1-\lambda}{p_{2}}$, that is $p \rightarrow\left\|S_{A}\right\|_{p}$ is a log-convex function of $\frac{1}{p}(p \geq 1)$.

Hence we can easily show Corollary 4.

\section{ACKNOWLEDGEMENT}

The author would like to express his hearty thanks to Professor T. Ando for his valuable suggestions and encouragement.

\section{REFERENCES}

1. T. Ando, R. A. Horn, and C. R. Johnson, The singular values of a Hadamard product: $a$ basic inequality, Linear and Multilinear Algebra 21 (1987), 345-365.

2. I. C. Gohberg and M. G. Krien, Introduction to the theory of linear nonselfadjoint operators, Amer. Math. Soc., Providence, RI, 1969. (translation)

3. G. H. Hardy, J. E. Littlewood, and G. Polya, Inequalities, 2nd ed., Cambridge Univ. Press, New York, 1952.

4. K. Okubo, Hölder-type norm inequalities for Schur product of matrices, Linear Algebra Appl. 91 (1987), 13-28.

5. S.-C. Ong, On the Schur multiplier norm of matrices, Linear Algebra Appl. 56 (1984), 45-55. 
6. V. I. Paulsen, Completely bounded maps and dilation, Pitman Res. Notes Math. Ser. no. $146,1986$.

7. I. Schur, Bemerkungen zur Theorie der Beschränkten Bilinearformen mit unendlich vielen Veränderlichen, J. Reine Angew. Math. 140 (1911), 1-28.

8. M. Walter, On the norm of a Schur product, Linear Algebra Appl. 79 (1986), 209-213.

Mathematics Laboratory, Sapporo College, Hokkaido University of Education, SapPORO 002, JAPAN 